\title{
Efficient eradication of potato viruses by induction of posttranscriptional gene silencing in transgenic potato
}

\author{
Alexandrova A.*, Nargilova R., Kryldakov R., Iskakov B., Karpova O. \\ M. Aitkhozhin Institute of Molecular Biology and Biochemistry, Almaty, Kazakhstan \\ *e-mail:alena_pisarenko@inbox.ru
}

Existing technologies for obtaining virus-free seed potatoes using methods of apical meristem cultivation, cryopreservation, chemo- and thermotherapy do not eliminate the possibility of subsequent viral infection of plants in the soil. Potato varieties with genetically engineered resistance against viral diseases are of particular importance.

Potato virus S (PVS, genus Carlavirus) is most widely spread in Kazakhstan. It has positive-sense single-stranded genomic RNA of 8535 nucleotides encoding six ORFs. Fragments of cDNA with complete coding sequence of $25 \mathrm{~K}$-protein in sense and antisense orientations were inserted at the $5^{\prime}$ and $3^{\prime}$ flank of cat 1 gene intron from Ricinus communis. Two recombinant cassettes [35S-CaMV-25KSense-intron-25KAsense-nos] or [35S-CaMV-25KAsense-intron-25KSense-nos] were cloned in pCAMBIA2300 binary vector under the control of 35S-CaMV promoter and nos-terminator. Expression of each cassette in planta leads to production of double-stranded RNAs that induce of RNAi mechanism against PVS.

Six potato cultivars initially containing viruses PVS, PVM, and PVY were transformed by aforementioned constructs. Regenerated plants were analyzed for presence of recombinant DNA constructs, as well as for their RNA-transcripts. Transgenic plants were grown in soil and further tested for the presence of viruses using the DAS-ELISA. Laboratory tests were performed every 30 days during 6 months. Five promising transgenic lines (TL) were selected. TL-61, TL-67 of "Dunyasha" variety and TL-103 of "Kormilitsa" variety showed negative response for PVM, which was detected before transformation. The TL-119, TL-336 lines of "Zeren" variety were free from the complex PVM+PVS+PVY viral infection.

Micro-tubers of transgenic potatoes were planted in the field with elevated viral background for trials in 2017-2018. Samples from these plants were collected for ELISA tests three times during the vegetative season. These analyses confirmed the results of laboratory tests: no potato viruses were detected in the samples. 